\title{
Desembarque e esforço de pesca da frota pesqueira comercial de Manicoré (Médio Rio Madeira), Amazonas, Brasil'
}

\author{
Renato Soares CARDOSO², Carlos Edwar de Carvalho FREITAS ${ }^{3}$
}

\begin{abstract}
RESUMO
A adequação das estratégias do pescador à sazonalidade existente na Amazônia atesta o nível de conhecimento tradicional em relação à ecologia dos peixes e às variações da pesca nesse ambiente, como ocorre em outras regiōes do Brasil. Nesse sentido, este trabalho teve como objetivo analisar a utilização dos ambientes de captura e a distribuição do esforço de pesca pela frota comercial em função do ciclo hidrológico. Foram coletados diariamente, entre junho de 2003 e maio de 2004, dados de desembarque e das expedições de pesca que ocorreram na região do Médio rio Madeira e submetidos à estatística descritiva. Os resultados mostraram que existiu uma tendência de aumento no esforço de pesca durante o período da enchente pelas embarcaçóes da frota local, para compensar a queda na produção capturada. Os barcos de pesca e canoas motorizadas apresentaram valores médios de CPUE de 22,9 e 20,6 kg/pescador*dia, respectivamente. Ficou evidenciada também a maior utilização dos ambientes igarapés e lagos pelos pescadores de canoas motorizadas e do rio pelos pescadores dos barcos.
\end{abstract}

PALAVRAS-ChaVE: Pesca interior, Locais de pesca, Esforço de pesca, Rio Madeira, Amazônia

\section{Landing and fishing effort of commercial fishing fleet of Manicoré city (Medium Madeira River), Amazonas, Brazil}

\section{ABSTRACT}

The adaptation of the strategies of the fisherman to the seasonality in the Amazonian attests the level of traditional ecological knowledge in relation to the fish and to the variations of the fishing in that environment, as it happens in other regions of Brazil. In that sense the present work aimed to analyze the use of the fishing grounds and the distribution of the fishing effort by the commercial fleet in relation to the hydrological cycle. Data of fishing landings and fishing expeditions of the Medium Madeira River region were collected daily, from June of 2003 until May of 2004, and submitted to the descriptive statistics. The results showed that there was a tendency towards an increase in the fishing effort during the rising water season for the vessels of the local fleet, to compensate for the decrease in the captured production. The fishing boats and motorized canoes presented medium values of CPUE 22.9 and $20.6 \mathrm{~kg} /$ fisherman*day, respectively. It was also shown that fishermen of motorized canoes utilized the stream and lake environments the most, where as boat fishermen relied mainly on the river environment.

KEYWORDS: Inland fishing, Fishing grounds, Fishing effort, Madeira River, Amazonian

\footnotetext{
1 Parte da Dissertação de Mestrado do primeiro autor do curso de Biologia de Água Doce e Pesca Interior do Instituto Nacional de Pesquisas da Amazônia - INPA.

2 Secretaria de Meio Ambiente e Desenvolvimento Sustentável - Prefeitura Municipal de Manicoré. Rua Marechal Deodoro da Fonseca, 364. Centro. CEP 69280-000. Manicoré, Amazonas, Brasil. Tel. (97) 3385-2170. e-mail: rsconpisci@yahoo.com.br

${ }^{3}$ Departamento de Ciências Pesqueiras - Universidade Federal do Amazonas. Avenida Rodrigo Otávio, 3000. Aleixo. CEP 69077-000. Manaus, Amazonas, Brasil. Tel. (92) 36474064. e-mail: cefreitas@ufam.edu.br
} 


\section{INTRODUÇÃO}

A adequação das estratégias do pescador frente à sazonalidade do ciclo hidrológico atesta o seu nível de conhecimento tradicional em relação à ecologia dos peixes e às variaçôes da pesca nesse ambiente, como ocorre em outras regiôes do Brasil. Isso pode ser comprovado pelo uso de vários apetrechos para a captura das mais diversas espécies de peixes que ocorrem em diferentes ambientes de pesca (Petrere Jr., 1978a; Batista et al., 2004).

Os ambientes de pesca existentes na região podem ser divididos em três principais: rios, lagos e igarapés. Eles apresentam subdivisões, dependendo da sazonalidade, como é o caso da floresta inundada adjacente ao rio (várzea) e aos lagos (igapós). Outros exemplos de ambiente sazonal são as praias e os poços, que são formados na calha do rio durante o período de seca e que também são utilizados para a captura de pescado.

A explotação desses ambientes é efetuada em conjunto pelos pescadores comerciais e de subsistência, com maior ou menor intensidade, dependendo da espécie-alvo e do apetrecho utilizado. Na região do Baixo rio Amazonas, a frota comercial explota principalmente a calha do rio na seca e os lagos durante a época da cheia (Cerdeira et al., 2000; Isaac et al., 2004), enquanto na região do Médio rio Solimões a frota local explota principalmente a calha do rio (Viana, 2004). Por outro lado, a pesca de subsistência é praticada principalmente nos lagos, na região do Baixo rio Amazonas e Médio rio Solimōes (Cerdeira et al., 2000).

$\mathrm{Na}$ região do rio Madeira, um dos maiores afluentes da margem direita do rio Amazonas em volume de água (Gibbs, 1965 apud Goulding, 1979), as frotas explotam a calha principal e a foz dos seus tributários (Goulding, 1979), porém os pesqueiros localizados nesse rio recebem também o esforço de pesca das frotas de diferentes Estados. A explotação na região do Baixo rio Madeira é efetuada pela frota do Pará e pela frota sediada em Manaus (Goulding, 1979). A porção Média e Alta são explotadas pelas frotas dos municípios amazonenses e pela frota do estado de Rondônia (Goulding, 1979; Cardoso, 2005). Nessa região, a frota pesqueira é formada por barcos e canoas motorizadas (Cardoso, 2005), que distribuem de maneira sistemática suas expediçóes para os locais de pesca.

Toda a logística que envolve a preparação das expedições de pesca no estado do Amazonas é organizada pelo encarregado da embarcação. A escolha do local de destino da expedição de pesca varia de acordo com a experiência do encarregado, que leva em consideração fatores como a facilidade de acesso ao local, o período do ciclo hidrológico e o custo total da expedição. Dentre as várias opções de locais existentes no raio de ação da frota, o escolhido será aquele que apresentar as melhores possibilidades de captura para aquele exato momento.
Como na região amazônica o ciclo hidrológico é um dos fatores que determina a captura de pescado, é importante para os gerenciadores do recurso, saber como os pescadores reagem às mudanças provocadas pelo mesmo nos ambientes de pesca $\mathrm{e}$ no comportamento das espécies explotadas comercialmente.

Nesse sentindo, o presente trabalho teve como objetivo, analisar a captura e a utilização dos ambientes pela frota pesqueira comercial do município de Manicoré, localizada na região do Médio rio Madeira, identificando os principais locais de pesca explotados e a distribuição do esforço entre os mesmos durante um ciclo hidrológico completo.

\section{MATERIAL E MÉTODOS}

Para efeito de análise, as capturas realizadas na praia, poço e paraná foram agrupados para o ambiente rio; as capturas realizadas no ambiente igapó foram agrupadas ao ambiente lago. Neste trabalho, o termo "embarcação de pesca" será utilizado para designar o conjunto formado pelo barco de pesca e canoa motorizada ou a remo.

\section{ÁREA DE ESTUDO}

O estudo abrangeu a região do Médio rio Madeira e teve como área focal o município de Manicoré, localizado na porção sudeste do Estado do Amazonas (Figura 1) que é um dos maiores municípios dessa região em termos populacionais e territoriais (IBGE, 2001). Uma descrição mais detalhada dos ambientes de pesca e das principais espécies explotadas na área de estudo pode ser encontrada em Cardoso (2005).

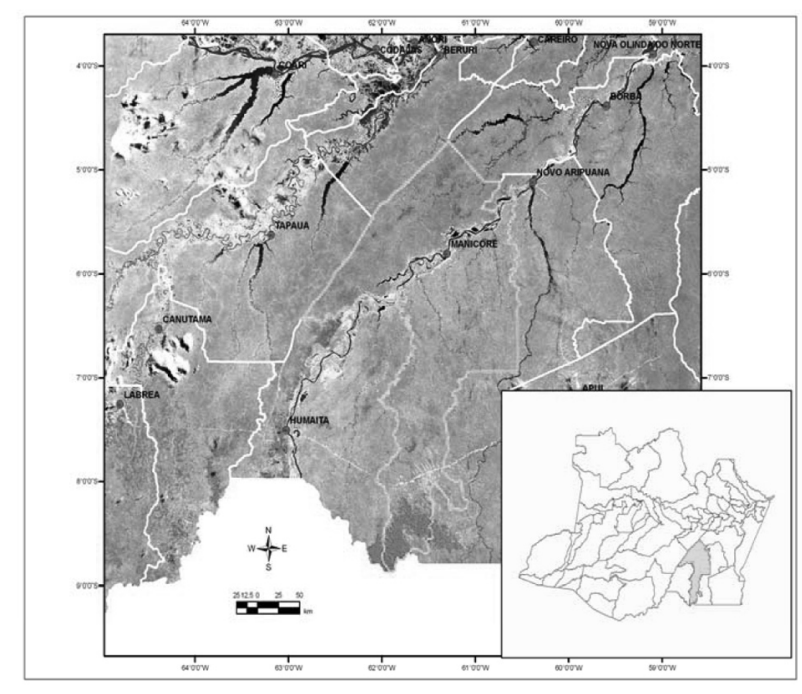

Figura 1 - Localização geográfica da área de estudo. 


\section{COLETA DOS DADOS}

Dados da produção de pescado foram coletados diariamente no mercado municipal, no período de junho de 2003 a maio de 2004, por intermédio de questionário estruturado, aplicado ao encarregado pela embarcação no momento do desembarque. As informações versaram sobre o local de pesca, número de pescadores na tripulação, data de partida e chegada da expedição, espécie (s) capturada (s), apetrechos utilizados e captura total $(\mathrm{kg})$.

\section{ANÁLISES EFETUADAS}

Os dados foram armazenados em planilhas eletrônicas, sendo em seguida, submetidos à estatística descritiva, para cálculo de freqüência, média e desvio padrão, como descreve Beiguelman (2002). Para relacionar o volume das capturas com o ciclo hidrológico anual, os dados da cota mensal do nível do rio Madeira (estação de Manicoré) foram obtidos junto a Agência Nacional de Águas (ANA).

Para o cálculo do esforço de pesca foi utilizada a unidade proposta por Petrere Jr. (1978b), que indicou como melhor unidade para a pesca na região amazônica o número de pescadores da tripulação multiplicado pelo número de dias de pesca. O esforço de pesca e a captura por unidade de esforço (CPUE) foram calculados separadamente para canoas e barcos de pesca.

\section{RESULTADOS}

A análise da série histórica do nível do rio para essa região do Médio rio Madeira (anos de 1994 a 2004) evidenciou que o ciclo hidrológico está assim dividido: período de vazante, durante os meses de maio a agosto; período de seca, nos meses de setembro e outubro; período de enchente, nos meses de novembro até fevereiro e período de cheia, nos meses de março e abril.

\section{A PRODUÇÃO LOCAL}

A produção total de pescado no período do estudo foi de 256,2 toneladas ( $\mathrm{t}$ ), com média de 21,3 t. mês $^{-1}$ (desvio padrão $=14,3$ t.mês $\left.{ }^{-1}\right)$, sendo o maior volume de produção em setembro (54,0 t) e o menor em março (3,9 t). O volume desembarcado por ambiente de captura variou em função do ciclo hidrológico e apresentou picos de produção nos meses de julho, setembro e abril (Figura 2), correspondente ao período de vazante, seca e cheia respectivamente.

A principal produção de pescado foi proveniente dos rios $(-149,7 \mathrm{t})$, sendo superior aos demais ambientes em onze meses do período estudado. A produção dos lagos $(-46,78 \mathrm{t})$ superou a produção dos rios somente no mês de maio. A produção dos igarapés $(-34,2 \mathrm{t})$ foi desembarcada principalmente nos meses da cheia e os desembarques em

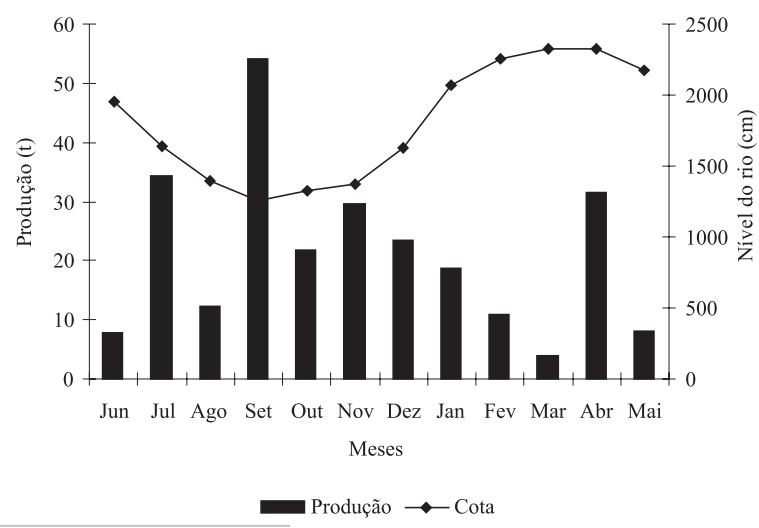

Figura 2 - Produção mensal de pescado desembarcado pela pesca comercial no município de Manicoré.

que não foi possível identificar o ambiente de origem $(-9,9$ t) foram maiores nos meses de setembro a novembro.

Três agentes de produção ligados à captura e comercialização do pescado efetuaram desembarques na área de estudo: barcos de pesca, canoas motorizadas e compradores. Os barcos de pesca apresentaram maior produção para todos os ambientes e na maioria dos meses do período estudado, sendo responsáveis por $55,0 \%$ de todo o pescado desembarcado, devido principalmente à sua maior capacidade de carga, enquanto canoas motorizadas $(23,2 \%)$ e compradores $(21,8 \%)$ tiveram volume percentual de desembarque semelhante. No entanto foram as canoas motorizadas que efetuaram o maior número de expedições de pesca (Tabela 1).

Tabela 1 - Número de desembarques efetuados por agente de produção.

\begin{tabular}{lcccc}
\hline \multicolumn{5}{c}{ Agente de produção } \\
\hline & Comprador & $\begin{array}{c}\text { Barco de } \\
\text { pesca }\end{array}$ & $\begin{array}{c}\text { Canoa } \\
\text { motorizada }\end{array}$ \\
\hline Mês/Ano & Número de & desembarques efetuados & Total \\
\hline Junho/2003 & 04 & 11 & 16 & 31 \\
Julho/2003 & 02 & 37 & 42 & 81 \\
\hline Agosto/2003 & 01 & 25 & 20 & 46 \\
\hline Setembro/2003 & 72 & 36 & 113 & 221 \\
\hline Outubro/2003 & 07 & 25 & 53 & 85 \\
\hline Novembro/2003 & 20 & 28 & 40 & 88 \\
\hline Dezembro/2003 & 14 & 23 & 31 & 68 \\
\hline Janeiro/2004 & 21 & 15 & 28 & 64 \\
\hline Fevereiro/2004 & 06 & 21 & 39 & 66 \\
\hline Março/2004 & 01 & 08 & 16 & 25 \\
Abril/2004 & 02 & 25 & 20 & 47 \\
Maio/2004 & 06 & 08 & 19 & 33 \\
\hline Total & $\mathbf{1 5 6}$ & $\mathbf{2 6 2}$ & $\mathbf{4 3 7}$ & $\mathbf{8 5 5}$ \\
\hline
\end{tabular}




\section{OS LOCAIS DE PESCA EXPLOTADOS}

A frota pesqueira explotou ao todo 52 locais de pesca entre igarapés (9), lagos (35) e rios (8). Os principais em termos de freqüência de expedições foram os pesqueiros localizados nos rios Madeira $(25,1 \%)$ e Manicoré $(16,6 \%)$ e no igarapé Matupiri (15,2\%).

Os igarapés mais importantes em frequiência de explotação foram: Matupiri $(85,0 \%)$, Jatuarana $(7,2 \%)$, Jacaré $(3,3 \%)$ e Baiua $(1,3 \%)$, sendo irrisória a freqüência de expediçōes para os outros cinco igarapés, explotados esporadicamente. $\mathrm{O}$ principal agente que efetuou a pesca no ambiente igarapé foi o pescador de canoa motorizada (63,4\% das expediçōes para esses locais). Entretanto, a quantidade de pescado desembarcado pelos barcos de pesca e por compradores, provindo desse ambiente, foi superior ao das canoas motorizadas durante os meses de junho e julho de 2003 e janeiro de 2004.

Dentre os lagos explotados, os lagos Boquerão (30,0\%) e Acará $(24,6 \%)$ foram os principais destinos das expediçôes de pesca, com destaque também para os lagos Matupirizinho $(8,4 \%)$, Jacaré $(4,4 \%)$ e Tapaiuna $(4,4 \%)$. Pescadores de canoas motorizadas foram os principais explotadores dos lagos $(66,0 \%)$, seguido pelos barcos $(28,6 \%)$ e compradores (5,4\%). Em apenas dois lagos (Boquerão e Acará), a freqüência de expediçōes dos barcos de pesca foi superior a dos outros agentes. O único lago a apresentar maior volume de desembarque efetuado por compradores foi o Matupirizinho, devido a esses agentes residirem nas comunidades localizadas nesse ambiente.

Dos agentes de produção que desembarcaram pescado dos lagos, os barcos de pesca apresentaram maior captura durante oito meses (junho-julho, outubro, dezembro-janeiro, março-maio), a produção das canoas superou a dos barcos e compradores em agosto-setembro, novembro e fevereiro. $\mathrm{O}$ mês de maior expressividade de desembarque dos compradores foi dezembro, porém no restante do período de estudo a produção desembarcada por este agente foi inexpressiva para este ambiente.

Dentre os rios explotados pela frota, o rio Madeira foi o principal em termos de freqüência de expedições $(51,1 \%)$, seguido pelos rios Manicoré (33,9\%) e Mataurá (6,9\%). Ocorreram também expediçōes para rios localizados em outros municípios da calha do rio Madeira, porém com baixa freqüência como no rio Aripuanã $(0,2 \%)$, município de Novo Aripuanã, região do Médio rio Madeira e no rio Canumã $(1,9 \%)$, município de Nova Olinda, regiāo do Baixo rio Madeira. As canoas motorizadas foram as que mais efetuaram expediçôes de pesca para os rios Madeira e Atininga, este último concentrando principalmente expedições de moradores das comunidades existentes na calha deste tributário.
Para os rios, a produção desembarcada pelos barcos de pesca, foi superior à dos outros agentes durante quase todo o período, exceção foi o mês de junho, quando foi registrado o menor desembarque por parte deste agente, sendo superado pela produção dos compradores. As canoas motorizadas apresentaram maior produção de pescado deste ambiente durante os meses de seca (setembro e outubro).

Parte dos desembarques de pescado efetuados por compradores não puderam ser identificados quanto a sua origem, principalmente no mês de setembro. Durante o mês de novembro e dezembro, ocorreu um aumento considerável de desembarque de pescado originado do município de Nova Olinda do Norte (Baixo rio Madeira), por parte dos compradores.

\section{ESFORÇO DE PESCA E CAPTURA POR UNIDADE DE ESFORÇO}

Os barcos de pesca apresentaram uma tendência de aumento no esforço de pesca de acordo com a elevação do nível do rio. O esforço de pesca aumentou durante o período da enchente e cheia, com um esforço maior no mês de março coincidindo com o mês de menor captura (Figura 2), e diminuiu durante o período da vazante e seca (Figura 3). As canoas motorizadas apresentaram aumento no esforço de pesca durante o período da enchente e cheia, atingindo um maior valor no mês de abril, sendo observado os menores valores no esforço durante os meses da seca, porém o menor valor de esforço foi observado no mês de junho, durante o período da vazante.

O rendimento pesqueiro mensal (CPUE) para os barcos de pesca variou entre $6,86 \mathrm{~kg} /$ pescador*dia no mês de março, durante o período da cheia e $45,19 \mathrm{~kg} /$ pescador* $^{*}$ dia no mês de setembro, durante o período da seca (Tabela 2). A CPUE para as canoas motorizadas apresentou valores entre $7,85 \mathrm{~kg} /$ pescador* dia no mês de março, durante o período da cheia e $38,02 \mathrm{~kg} /$ pescador*dia no mês de agosto, durante o período da vazante.

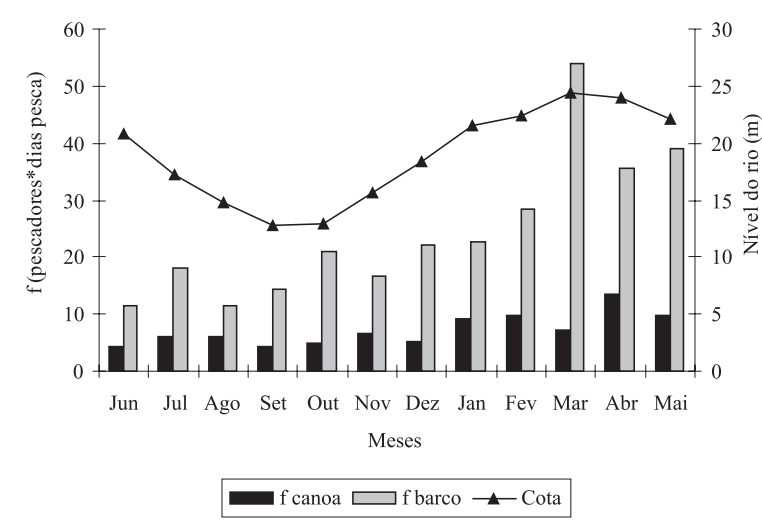

Figura 3 - Esforço de pesca (f) da frota pesqueira de Manicoré. 
Tabela 2 - Captura por Unidade de Esforço (CPUE) e captura mensal para a frota pesqueira de Manicoré.

\begin{tabular}{lcccc}
\hline & \multicolumn{2}{c}{ CPUE } & \multicolumn{2}{c}{ Captura média $(\mathrm{kg})$} \\
\cline { 2 - 5 } Mês & $\begin{array}{c}\text { Canoa } \\
\text { motorizada }\end{array}$ & $\begin{array}{c}\text { Barco de } \\
\text { pesca }\end{array}$ & $\begin{array}{c}\text { Canoa } \\
\text { motorizada }\end{array}$ & $\begin{array}{c}\text { Barco de } \\
\text { pesca }\end{array}$ \\
\hline Junho & 18,65 & 17,70 & 78,33 & 205,27 \\
Julho & 21,86 & 41,78 & 130,51 & 761,24 \\
Agosto & 38,04 & 27,54 & 226,72 & 318,58 \\
Setembro & 27,90 & 45,17 & 123,03 & 650,46 \\
\hline Outubro & 33,63 & 23,07 & 160,76 & 481,62 \\
Novembro & 25,20 & 27,46 & 166,56 & 455,04 \\
Dezembro & 17,98 & 23,23 & 95,11 & 514,61 \\
Janeiro & 16,42 & 12,57 & 151,85 & 286,07 \\
Fevereiro & 8,65 & 11,23 & 84,75 & 319,43 \\
Março & 7,85 & 6,86 & 56,94 & 370,88 \\
Abril & 18,60 & 28,09 & 249,58 & 999,84 \\
Maio & 14,06 & 10,61 & 138,38 & 413,3 \\
\hline
\end{tabular}

\section{DISCUSSÃO}

Seguindo a tendência apresentada em algumas microrregiōes da Amazônia (Barthem, 1999; Batista \& Petrere Jr., 2003; Isaac et al., 2004; Viana, 2004), as pescarias no município foram direcionadas com maior freqüência para os rios, principalmente para o rio Madeira, o que denota a importância desse ambiente para a pesca comercial local, sobretudo para os barcos, que foram responsáveis pela maior parte das capturas. Goulding (1979) analisando os desembarques de pescado na região do Alto rio Madeira, já apontava para a tendência de utilização da foz dos tributários do rio Madeira, como principal local de captura da frota comercial, principalmente durante a migração reprodutiva.

O aparecimento do rio Manicoré como segundo pesqueiro de maior utilização, se deve em grande parte à sua localização próxima a sede do município. Isso facilita os deslocamentos tanto de barcos quanto de canoas motorizadas até os ambientes de pesca existentes nesse tributário. Conforme indicaram Cardoso \& Freitas (2006), as pescarias efetuadas nos pesqueiros localizados no rio Manicoré foram menos onerosas, pois não necessitaram de gelo e consumiram pouco combustível.

Isso é atrativo aos pescadores, pois diminui consideravelmente os custos das expedições, aumentando teoricamente, a renda por viagem de pesca. Isaac et al. (2004), analisando os desembarques da região do Baixo rio Amazonas, identificaram que as pescarias foram efetuadas em maior freqüência nos pesqueiros próximos à sede do município de origem da frota, porém não destacaram se isso foi devido ao baixo custo da expedição de pesca ou à maior produtividade dos pesqueiros.
Outra explicação para a grande freqüência de expedições para o rio Manicoré durante o período de cheia, é que o mesmo se conecta ao rio Madeira, por um "furo" denominado Varador Moreira (Cardoso, 2005). Esse pesqueiro é bastante freqüentado pelos pescadores de barco devido a grande produtividade das pescarias de jatuarana (Brycon spp) e jaraqui (Semaprochilodus spp) durante os meses de abril e maio (Cardoso, 2005). Esse fator foi a causa principal do pico de captura observado no mês de abril.

Visto que os pescadores de canoas motorizadas desta região, empregam principalmente apetrechos de captura passivos, como as malhadeiras (Cardoso, 2005), o padrão de utilização de lagos e igarapés durante o período da enchente e cheia, foi o esperado. Esses ambientes não apresentam correnteza, o que facilita a utilização desses apetrechos (Barthem \& Fabré, 2004) e a captura ocorre principalmente nos igapós formados pela expansão desses corpos aquáticos, quando os peixes procuram se alimentar nesses locais.

Vale ressaltar que foi nesse período (enchente e cheia), que as capturas provenientes dos lagos superaram a dos outros ambientes, provavelmente isso tenha ocorrido devido a pouca frequêencia de cardumes nos rios, e também em decorrência do defeso, imposto pelo órgão de regulamentação, que não permite a captura de várias espécies e a utilização de determinados rios para a pesca comercial (Vicentini, 2005).

Os principais lagos em produção de pescado e freqüência de expedições de pesca, apresentaram diferenças quanto aos seus principais explotadores. O Boquerão que é um lago de banco de ferradura, formado através do isolamento de meandros pelo processo de erosão e sedimentação das margens (Esteves, 1998), localizado a jusante da sede do município, foi explotado principalmente por barcos de pesca que utilizam malhadeiras para a captura de pescado. Essa maior frequiência de explotação pode ser atribuída à sua proximidade da calha principal do rio Madeira, o que facilita o acesso dos barcos. O lago Acará, inversamente ao que ocorreu com o lago Boquerão, foi explotado principalmente por canoas motorizadas, se tornando um dos principais locais para a captura de pescado durante os meses de cheia.

O padrão de utilização observado para as canoas motorizadas evidenciou que as mesmas explotaram os lagos durante a enchente, cheia e início da vazante. Entretanto suas capturas foram orientadas principalmente, para a calha do rio Madeira durante o final da vazante e seca, quando seus deslocamentos foram menores, permanecendo nas proximidades da sede do município em pescarias de, no máximo, um dia de duração (Cardoso, 2005).

Os igarapés apresentaram menor importância em termos de produção pesqueira do que os lagos. O principal igarapé em frequiência de explotação e produção desembarcada 
(igarapé Matupiri) é também uma área de conflito de uso entre os pescadores comerciais de canoas motorizadas e os moradores das comunidades locais. Esse igarapé dá acesso à reserva extrativista Matupiri Grande, onde a pesca de caráter comercial é proibida pela Portaria 496/96 do IBAMA, sendo liberada apenas no mês de março, período máximo da cheia, quando as capturas são relativamente menores e não é um local atrativo para os pescadores das canoas motorizadas. Esse conflito não é o único nessa região (Cardoso, 2005), pois alguns ambientes de pesca, principalmente igarapés, estão sob controle ou são de "propriedade" de algum agente de produção, sendo os demais pescadores impedidos de pescar, mesmo com a legislação pesqueira brasileira classificando os ambientes aquáticos como de livre acesso.

$\mathrm{O}$ aumento no esforço de pesca efetuado pelos pescadores de canoa motorizada nos períodos de enchente e cheia ocorreu, provavelmente, devido à dispersão dos cardumes nas áreas alagadas da várzea e nos igapós formados nas áreas de entorno dos lagos, dificultando a captura de pescado. Isso pode ser comprovado quando se observa o peso médio no desembarque neste período para esse agente de pesca. Esse padrão também foi encontrado por Barthem (1999) para pescadores de canoas motorizadas que desembarcaram pescado no município de Tefé, na região do Médio rio Solimões. Esses pescadores também são encontrados na região do Baixo Amazonas na cidade de Santarém, porém os dados de desembarque de pescado para essa área não mencionam a quantidade de pescado desembarcado por este agente, muito menos faz referência ao esforço de pesca empregado por estes pescadores.

Convém salientar que consideramos os resultados deste trabalho preliminares e ainda não conclusivos, sendo necessária a utilização de uma série histórica maior de dados para corroborar as tendências apresentadas. Entretanto, os resultados podem ser utilizados como um indicativo para a tomada de decisóes concernentes ao manejo pesqueiro desta região do rio Madeira, principalmente no que diz respeito ao fechamento anual de locais para a pesca comercial. Outro fator a ser levado em consideração pelos gerenciadores é que embora a maioria dos pescadores comerciais de outras regióes tenha conhecimento de que os ambientes aquáticos sejam de livre acesso, nesta região ainda existe a necessidade que os explotadores do recurso sejam melhor informados sobre a situação de ilegalidade da proibição do uso desses locais por parte de particulares.

\section{CONCLUSÕES}

Observando o desembarque de pescado e a utilização dos ambientes de pesca pela frota comercial no município de Manicoré durante o ciclo hidrológico estudado nos permitiu concluir que:

a) Existiu uma separação na distribuição do esforço pesqueiro da frota pelos ambientes, com os barcos de pesca explotando principalmente os rios, enquanto canoas motorizadas explotaram lagos e igarapés e os compradores mostraram-se mais generalistas;

b) Durante o período da enchente e cheia os pescadores de canoas motorizadas e barcos aumentaram o esforço de pesca para compensar o menor rendimento desses períodos menos produtivos;

c) As canoas motorizadas realizaram mais expediçōes de pesca, porém os barcos foram os responsáveis pela captura da maior quantidade do pescado desembarcado no município.

\section{AGRADECIMENTOS}

Os autores gostariam de agradecer ao $\mathrm{CNPq}$ pelo financiamento da pesquisa, aos pescadores de Manicoré pelo fornecimento dos dados, à colônia de pescadores Z-20 pelo apoio durante o trabalho, aos revisores pelas críticas e sugestōes e a Srta. Marijara Santos pela ajuda na coleta das informações.

\section{BIBLIOGRAFIA CITADA}

Barthem, R.B. 1999. A pesca comercial no médio Solimões e sua interação com a reserva Mamirauá. In: Queiroz, H.L.; Crampton, W.G.R. (ed.). Estratégias para manejo de recursos pesqueiros em Mamirauá. Sociedade Civil Mamirauá. MCT-CNPq. p. 72107.

Barthem, R.B.; Fabré, N.N. 2004. Biologia e diversidade dos recursos pesqueiros da Amazônia. In: Ruffino, M.L. (coord.). A pesca e os recursos pesqueiros na Amazônia brasileira. IBAMA/ PROVÁRZEA. p. 17-62.

Batista, V.S.; Petrere Jr., M. 2003. Characterization of the commercial fish production landed at Manaus, Amazonas State, Brazil. Acta Amazonica, 33(1): 53-66.

Batista, V.S.; Isaac, V.J.; Viana, J.P. 2004. Exploração e manejo dos recursos pesqueiros da Amazônia. In: Ruffino, M.L. (coord.). A pesca e os recursos pesqueiros na Amazônia brasileira. IBAMA/ PROVÁRZEA. p. 63-152.

Beiguelman, B. 2002. Curso prático de bioestatística. $5^{\mathrm{a}}$. ed. Fundação de Pesquisas Científicas de Ribeirão Preto - FUNPEC. Ribeirão Preto, 274pp. 
Cardoso, R.S. 2005. A pesca comercial no município de Manicoré (rio Madeira), Amazonas, Brasil. Dissertação de Mestrado, Instituto Nacional de Pesquisas da Amazônia/Fundação Universidade Federal do Amazonas, Manaus, Amazonas. 149pp.

Cardoso, R.S.; Freitas, C.E.C. 2006. A composição dos custos de armação e a renda das expedições de pesca da frota pesqueira artesanal da região do Médio rio Madeira, Amazonas, Brasil. Acta Amazonica, 36(4): 529-524.

Cerdeira, R.G.P.; Ruffino, M.L.; Isaac, V.J. 2000. Fish catch among riverside communities around Lago Grande de Monte Alegre, Lower Amazon, Brazil. Fisheries Management and Ecology, 7: 355-374.

Esteves, F.A. (1998). Fundamentos de limnologia. 2a . Ed. Interciência. Rio de Janeiro. 602pp.

Goulding, M. 1979. A ecologia da pesca no rio Madeira. CNPq/ INPA. Manaus. 172p.

IBGE. 2001. Censo demográfico 2000: Características da população e dos domicílios. IBGE. Rio de Janeiro. 519pp.

Isaac, V.J.; Silva, C.O.; Ruffino, M.L. 2004. A pesca no Baixo Amazonas. In: Ruffino, M.L. (coord.). A pesca e os recursos pesqueiros na Amazônia brasileira. IBAMA/PROVÁRZEA. p. 185-211.
Petrere Jr., M. 1978a. Pesca e esforço de pesca no Estado do Amazonas. II. Locais, aparelhos de captura e estatística de desembarque. Acta Amazonica, 8(supl. 2): 1-54.

Petrere Jr., M. 1978b. Pesca e esforço de pesca no Estado do Amazonas. I. Esforço e captura por unidade de esforço. Acta Amazonica, 8(Suplemento 3): 439-454.

Viana, J.P. 2004. A pesca no Médio Solimões. In: Ruffino, M.L. (coord.). A pesca e os recursos pesqueiros na Amazônia brasileira. IBAMA/PROVÁRZEA. p. 245-268.

Vicentini, R.N. 2005. Avaliação dos efeitos de medidas de manejo na pesca comercial efetuada na Amazônia Central. Dissertação de Mestrado, Instituto Nacional de Pesquisas da Amazônia/ Fundação Universidade Federal do Amazonas, Manaus, Amazonas. 96pp.

Recebido em 13/04/2007

Aceito em 18/09/2007 
